\title{
Handlungssicherheit in alltäglichen Begegnungen \\ Die Freie Wohlfahrtspflege bietet auch spezialisierte Qualifizierungen im sozialen Ehrenamt
}

HANNES JÄHNERT

Hannes Jähnert ist Referent zur Förderung sozialen Ehrenamts beim Generalsekretariat des Deutschen Roten Kreuzes in Berlin.

www.drk.de

\author{
Anhand des Curriculums des Deutschen Roten Kreuzes \\ für Ehrenamtliche in Besuchsdiensten kann gezeigt \\ werden, wie die Anerkennung bürgerschaftlichen \\ Engagements durch Qualifizierung im sozialen Ehrenamt \\ gelingt und welch bedeutsame Rolle dabei die \\ Ehrenamtskoordination spielt.
}

Die Qualifizierung im sozialen Ehrenamt kann der Anerkennung und Bindung sozial engagierter Ehrenamtlicher dienen. Dafür müssen den Ehrenamtlichen sowohl typische als auch besondere Qualifizierungen adäquat angeboten werden. Am Beispiel des bundeseinheitlichen Curriculums für ehrenamtliche Besuchsdienste im Deutschen Roten Kreuz wird in diesem Beitrag erläutert, wie Qualifizierung im sozialen Ehrenamt gelingen kann.

Mit sozialem Ehrenamt werden in der Regel organisierte Formen des freiwilligen Engagements bezeichnet, bei denen sich die Ehrenamtlichen in alltäglichen Settings hilfebedürftigen Menschen zuwenden. Grob unterscheiden lassen sich dabei zwei Typen: der Aufbau und die Pflege von Strukturen für Hilfebedürftige wie beispielsweise Kleiderläden und Tafeln sowie der Aufbau und die Pflege von Beziehungen zwischen Ehrenamtlichen und Hilfeempfängern wie beispielsweise Vorlesepatenschaften und Besuchsdienste. Beim sozialen Ehrenamt - und ganz besonders dem zweiten Typus - geht es dementsprechend weniger um das Einbringen konkreter fachlicher Kompetenzen, sondern vielmehr darum, eigene freie Zeit für Beziehungsaufbau und Beziehungspflege zu den Adressaten des Engagements einzusetzen.

Damit stellt sich freilich die Frage, inwiefern in sozialen Ehrenämtern eigentlich Qualifizierungen notwendig sind. Es geht ja gerade darum, alltägliche
Beziehungen - nicht professionellen Settings - zu gestalten. Für das Engagement in den Besuchsdiensten des Deutschen Roten Kreuzes (DRK) wurde diese Frage wie folgt beantwortet:

»Die Qualifizierung im ehrenamtlichen Besuchsdienst soll die Engagierten bedarfsgerecht zur Handlungssicherheit im DRK-Ehrenamt befähigen. Dies kann nicht in einer einmaligen Ausbildung oder Schulung geleistet werden. Die bedarfsgerechte Befähigung zur Handlungssicherheit ist ein Prozess, der das Engagement im ehrenamtlichen Besuchsdienst begleitet. (Deutsches Rotes Kreuz 2014: Seite 4)

Qualifizierung in diesem Sinne meint eine Form der Anerkennung sozialen Ehrenamts als unterstützenswerter Gestaltungsprozess von Beziehungen mit dem Ziel vor Leiden - beispielsweise durch Vereinsamung - zu bewahren oder sie zu lindern. Grundlage dafür sind die Bedürfnisse der Ehrenamtlichen wie der Adressaten ihres Engagements.

\section{Typische und besondere Qualifizierungsangebote im Ehrenamt}

Bei der Erarbeitung des Curriculums für ehrenamtliche Besuchsdienste im Deutschen Roten Kreuz stellten sich die Fragen, wie verbandsweite Standards für die Qualifizierung Ehrenamtlicher im Besuchsdienst mit dem Anspruch 
individueller Bedarfsgerechtigkeit von Qualifizierungsangeboten im sozialen Ehrenamt vereinbart werden können und wie sich diese Qualifizierungsangebote in der Praxis gestalten lassen.

Ausgehend von bestehenden Qualifikationsangeboten in zahlreichen, teilweise schon mehr als zehn Jahren bestehenden Besuchsdiensten und in Zusammenarbeit mit Expertinnen und Experten aus dem Verband wurde ein Praxisleitfaden für Ehrenamtskoordinationen und verantwortliche Leitungen von Besuchsdiensten im Deutschen Roten Kreuz erarbeitet, in dessen Zentrum fünf Lernfelder stehen: »Praktische Hilfen", "Sozialkompetenzen", »Psycho-Soziales «, »Rechtliches « und »DRK-Internes«.

\section{Typische Qualifizierungsangebote}

Die ersten vier der genannten Lernfelder des Curriculums schließen unmittelbar an die Tätigkeit in Besuchsdiensten an und können dementsprechend als typische Qualifizierungsangebote im sozialen Ehrenamt verstanden werden.

In den Lernfeldern »Praktische Hilfen " und "Sozialkompetenzen " werden vielerlei Inhalte vermittelt, die für die praktische Tätigkeit im ehrenamtlichen Besuchsdienst nützlich sind. Beispielhaft zu nennen wäre hier der Umgang mit dem Rollstuhl oder anderen Hilfsmitteln, die im pflegerischen Kontext zum Einsatz kommen, sowie diverse Techniken der Gesprächsführung, die von Ehrenamtlichen angewandt werden können, um »das Eis zu brechen«.

Im Lernfeld »Psycho-Soziales" werden unterschiedliche Inhalte zur Selbstfürsorge und Reflexion des Engagements vermittelt. $Z u$ nennen wäre hier beispielhaft die Grenzen des persönlichen Engagements. Im Lernfeld »Rechtliches « schließlich werden Inhalte zu rechtlichen Aspekten des Engagements wie Unfallund Haftpflichtversicherung, Schweigepflicht und Datenschutz vermittelt.

\section{Besondere Qualifizierungsangebote}

Im fünften Lernfeld - »DRK-Internes «werden Inhalte vermittelt, die auf den weiteren Rahmen eines DRK-Ehrenamtes zielen und insofern als besondere Qualifizierungsangebote verstanden werden können.
Das Deutsche Rote Kreuz hat besondere Strukturen wie seine fünf ehrenamtlichen Gemeinschaften - Bergwacht, Wasserwacht, Bereitschaften, Jugendrotkreuz und Wohlfahrts- und Sozialarbeit - und spezielle Aufgaben, wie die Verbreitung des humanitären Völkerrechts oder die Resilienzsteigerung der deutschen Bevölkerung und Infrastruktur.

Ehrenamtlichen diese Besonderheiten zu vermitteln, ist aus der Perspektive des Verbandes und insbesondere vor dem Hintergrund der Bemühungen, Ehrenamtliche an den Verband zu binden und sie in ihren Potentialen weiterzuentwickeln (»Ehrenamtskarrieren«) sehr

\section{Rolle der Ehrenamtskoordination}

Die Ehrenamtskoordination bildet das Scharnier zwischen unterschiedlichen Anforderungen und Ansprüchen der Ehrenamtlichen und der Organisation. Sie vermittelt zwischen unterschiedlichen Standpunkten und ist bemüht, alle relevanten Personen- und Interessengruppen «im Boot« zu haben. In das Curriculum für ehrenamtliche Besuchsdienste ist dieses Rollenverständnis der Ehrenamtskoordination eingeflossen. So wird explizit darauf verwiesen, in die Konzeptionierung und Planung der Qualifizierungsangebote alle relevanten Personen mit einzubeziehen. Dabei

\section{"Ehrenamtskoordination ist Mittler} zwischen unterschiedlichen Standpunkten, Netzwerkknotenpunkt und Promotor

\section{ehrenamtlichen Engagements innerhalb und außerhalb der Organisation«}

sinnvoll. Diese besonderen Qualifizierungsangebote tragen entsprechend zur Handlungssicherheit im Ehrenamt des Deutschen Roten Kreuzes bei.

\section{Niedrigschwellige Einstiege und die Rolle der Ehrenamtskoordination}

Anders als die Inhalte typischer Qualifizierungsangebote, tangieren die besonderen Aufgaben und Strukturen des Deutschen Roten Kreuzes Ehrenamtliche in Besuchsdiensten zunächst nur am Rande. Aus der Perspektive sozial engagierter Ehrenamtlicher, die sich ganz praktisch Hilfebedürftigen zuwenden und ihnen alltägliche Beziehungen anbieten wollen, werden die entsprechenden Angebote - zumindest bei Aufnahme der Tätigkeit - deshalb recht häufig als weniger bedarfsgerecht oder gar als aufoktroyiert empfunden. Hier wird der klassische Widerspruch zwischen den Bedürfnissen und Motivlagen Ehrenamtlicher und den Ansprüchen von Organisationen wie dem Deutschen Roten Kreuz deutlich, zwischen denen die Ehrenamtskoordination im Verband vermittelt. geht es einerseits darum, möglichst viel Expertise in den Planungsprozess einzubeziehen, und andererseits darum, für das soziale Ehrenamt in der Einrichtung oder Organisation zu werben - es geht um Netzwerkarbeit und (internes) Marketing; ebenfalls wesentliche Bestandteile der Ehrenamtskoordination.

Neben ihrer Funktion als Mittler zwischen unterschiedlichen Standpunkten, Netzwerkknotenpunkt und Promotor ehrenamtlichen Engagements innerhalb und außerhalb der Organisation, ist die Ehrenamtskoordination vor allem Ansprechpartner und Wegbegleiter für die Ehrenamtlichen. Dementsprechend sollte die Ehrenamtskoordination den immer individuell verlaufenden Prozess des sozialen Engagements mit Qualifizierungsangeboten begleiten und unterstützen.

\section{Niedrigschwellige Einstiege}

Für den Anfang dieses QualifizierungsProzesses werden im Curriculum für ehrenamtliche Besuchsdienste niedrigschwellige Einführungen in die genannten fünf Lernfelder vorgeschlagen. Beschrieben werden lediglich Basisin- 
halte, auf denen im weiteren Verlauf des Engagements aufgebaut werden muss. Die Erfahrungen mit sozialem Ehrenamt im Deutschen Roten Kreuz zeigen, dass Qualifizierungsangebote während eines Engagements dann besonders gut angenommen werden, wenn der Nutzen für die praktische Tätigkeit der Ehrenamtlichen klar erkennbar ist oder Ehrenamtliche auf eigene Wissenslücken aufmerksam werden. Insofern geht es bei den niedrigschwelligen Einführungen insbesondere darum, gezielt Qualifizierungsbedarfe zu wecken.

Dass dies funktioniert, zeigte das »EASI «-Projekt aus der DRK-Ortsvereinigung Raunheim-Kelsterbach (DRKLandesverband Hessen). Ausgehend von der Feststellung, dass einem Gros der älteren Generation die Kompetenz für erste Hilfe bei Notfällen fehlt, diese aber eher schwer zu motivieren ist, einen entsprechenden Kurs zu besuchen, wurde im Rahmen eines kurzen, einführenden Workshops zunächst nur die Basis der ersten Hilfe vermittelt, beispielsweise Kommunikation mit der Rettungsstelle, Vorbereitung des Eintreffens der Sanitäter und Basismaßnahmen der ersten Hilfe. Die Rückmeldungen der Teilnehmenden zeigten, dass viele auf eigene Wissenslücken aufmerksam wurden und sich nunmehr motiviert sahen, einen vollständigen Erste-Hilfe-Kurs zu absolvieren.

\section{Die Lernfeldmatrix - ein Werkzeug für die Ehrenamtskoordination}

Das Aufgabenspektrum der Ehrenamtskoordination ist, wie oben deutlich wurde, recht umfangreich - die zur Verfügung stehenden zeitlichen Ressourcen sind es dagegen nicht. Diese kritikwürdige Diskrepanz erklärt, warum sich einfach zu handhabende Werkzeuge in der Ehrenamtskoordination so großer Beliebtheit erfreuen. Sie bieten die Möglichkeit der Zeitnot bei der Begleitung Ehrenamtlicher mit Standards zu begegnen, die auch dem Ressourcenmanagement sowie der Qualitätssicherung und Qualitätsentwicklung zuträglich sind und darüber hinaus Mobilität insbesondere den Wechsel von Ehrenamtlichen aus einer Gliederung in eine andere - ermöglichen. (Im Anschluss an die Studien Hartmut Rosas zur Beschleunigung in der Moderne meint
"Zeitnot « in diesem Zusammenhang nicht etwa die faktisch fehlende Zeit für die Ehrenamtskoordination, sondern viel eher ein diffuses Gefühl rasender Zeit, vor dessen Hintergrund die Entscheidung für »smarte Tools« - Werkzeuge, deren Benutzung sich problemlos dazwischen schieben lässt - "alternativlos « erscheint.)

Auch für das Curriculum für ehrenamtliche Besuchsdienste im Deutschen Roten Kreuz wurde ein solches Werkzeug entwickelt. Die Lernfeldmatrix teilt die fünf oben genannten Lernfelder in »Informationen vorweg ", »Basiswissen «, »Vertiefungswissen « und »Ergänzungswissen« ein.

Die ersten zwei Kategorien werden im Curriculum behandelt. Bei »Informationen vorweg " handelt es sich um alles, was Ehrenamtliche wissen sollten, bevor sie ihr Engagement aufnehmen. Dazu zählt unter anderem das Engagementprofil, die Grundsätze und Leitlinien des Deutschen Roten Kreuzes, die Datenschutz- und Verschwiegenheitserklärung sowie eine kurze Darstellung zum Verhalten beim Besuch (»Verhaltenskodex«). Diese Informationen können für Basisqualifizierungen wieder aufgenommen werden, wobei es hier - wie gesagt insbesondere darum geht, Neugier an Mehr zu wecken.

Der Nutzen der Lernfeldmatrix besteht nun einerseits darin, bei der Planung bedarfsgerechter Qualifizierungsangebote keine blinden Flecken entstehen zu lassen. So gibt es - das zeigte die Analyse der vorliegenden Materialien bei der Erarbeitung des Curriculums - zahlreiche typische Qualifizierungsangebote für das soziale Ehrenamt im Besuchsdienst. Die besonderen Qualifizierungsangebote dagegen werden oft nur angeschnitten und selten vertieft.

Andererseits besteht der Nutzen der Lernfeldmatrix darin, den individuellen Qualifizierungsverlauf einzelner Ehrenamtlicher abzubilden. Die Matrix ist dementsprechend ein Dokumentationswerkzeug, auf dessen Grundlage - beispielsweise in Feedback- oder Entwicklungsgesprächen - gemeinsam mit den Ehrenamtlichen vertiefende und weiterführende Qualifizierungen identifiziert werden können, die an deren jeweils aktuellen Bedürfnisse, Interessen und Neigungen anschließen und auf vorausgegangene Qualifizierungen aufbauen.

\section{Schlusswort}

Die Qualifizierung im Ehrenamt hat im Deutschen Roten Kreuz lange Tradition. Qualifizierungsangebote für sozial engagierte Ehrenamtliche allerdings unterscheiden sich von denen für Ehrenamtliche in anderen Engagementfeldern wie beispielsweise dem Rettungsdienst. Umfassende Schulungen, die auf alle möglichen Eventualitäten vorbereiten sollen, sind hier wenig zielführend, weil sich das soziale Ehrenamt insbesondere durch die alltägliche Hinwendung zu Hilfebedürftigen auszeichnet.

Wie im sozialen Ehrenamt besondere Qualifizierungsangebote, die auf den weiteren Rahmen des Engagements zielen, so gestaltet werden können, dass sie von Ehrenamtlichen als hilfreich und nützlich angesehen werden, ist eine Frage, mit der sich die Ehrenamtskoordination in Verbänden wie dem Deutschen Roten Kreuz auseinander setzen muss.

Wenn sich die Qualifizierung im sozialen Ehrenamt nicht allein auf die Vermittlung von Handwerkszeug für die praktische Tätigkeit beschränkt, sondern auch zur Handlungssicherheit im weiteren Rahmen des Engagements beiträgt, birgt sie viel Potenzial für Ehrenamtskarrieren und damit der weiteren Stärkung des sozialen Ehrenamts in Wohlfahrtsverbänden wie dem Deutschen Roten Kreuz.

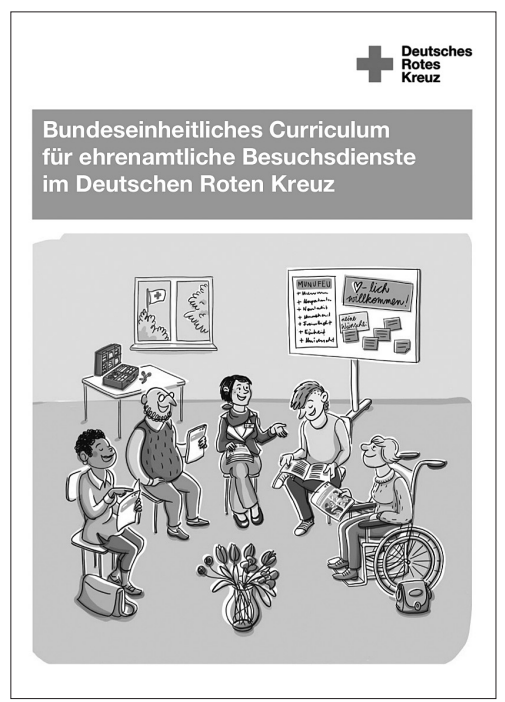

Das Curriculum für Besuchsdienste im Deutschen Roten Kreuz steht als kostenlose Broschüre im Internet zur Verfügung.

www.drk-wohlfahrt.de 